\title{
REVIEW
}

\section{Stribild, a Single Tablet Regimen for the Treatment of HIV Disease}

Cynthia Brinson

To view enhanced content go to www.combitherapy-open.com

Received: January 11, 2013 / Published online: March 26, 2013

(c) The Author(s) 2013. This article is published with open access at Springerlink.com

\section{ABSTRACT}

Introduction: In August 2012, the US Food and Drug Administration (FDA) approved a new single tablet once-a-day therapy for treatmentnaïve HIV patients. The new tablet contains emtricitabine and tenofovir disoproxil fumarate as well as elvitegravir and cobicistat, a pharmacokinetic enhancer which prolongs the effect of elvitegravir. The new tablet (EVG/ COBI/FTC/TDF), known as Stribild ${ }^{\circledR}$ (Gilead Sciences, Foster City, CA, USA), is now the only FDA-approved single-tablet, once-daily, HIV medication that is composed of an integrase-inhibitor-based regimen.

Methods: Stribild has been tested in two randomized double-blind phase 3 clinical trials with 1,408 patients who had not been previously treated for HIV. In one trial, Stribild

C. Brinson ( $\square)$

Central Texas Clinical Research, Austin, TX, USA

e-mail: cbrinson@ctcrcorp.com

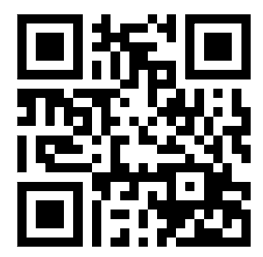

Enhanced content for this article is available on the journal web site: www.combitherapy-open.com was compared to the single-tablet regimen gold standard medication known as Atripla ${ }^{\circledR}$ (Gilead Sciences, Foster City, CA, USA) that contains efavirenz, emtricitabine and tenofovir disoproxil fumarate (EFV/FTC/TDF). In the second clinical trial, Stribild was compared to another preferred treatment regimen of ritonavir-boosted atazanavir (ATV/RTV) with coformulated emtricitabine and tenofovir disoproxil fumarate (FTC/TDF, marketed as Truvada $^{\circledR}$; Gilead Sciences, Foster City, CA, USA).

Results: The outcomes of the two recently published trials at 48 weeks indicated that Stribild was noninferior to both of the standard treatment regimens in controlling viral load. In the Stribild versus Atripla trial, 305 of 348 patients $(87.6 \%)$ on Stribild versus 296 of 352 patients $(84.1 \%)$ on Atripla had an HIV ribonucleic acid (RNA) concentration of $<50$ copies/mL at week 48 . In the Stribild versus ATV/RTV with Truvada trial, 316 of 353 patients (89.5\%) on Stribild versus 308 of 355 patients $(86.8 \%)$ on Atripla had an HIV RNA concentration of $<50$ copies $/ \mathrm{mL}$ at 48 weeks.

Conclusion: Stribild had a favorable safety profile in the two recently published 
randomized, double-blind, phase 3 clinical trials. With the approval of Stribild, clinicians now have more flexibility in prescribing singletablet regimens for patients.

Keywords: Antiretroviral;

Cobicistat;

Dyslipidemia; Emtricitabine; Hepatitis; HIV; Integrase-inhibitor; Protease-inhibitor; Singletablet therapy; Stribild; Tenofovir disoproxil fumarate

\section{INTRODUCTION}

Since the mid-1990s, a number of advances have improved treatment efficacy as well as ease of administration in HIV. The current standard of care for treatment-naïve patients is a combination of at least three active medications chosen from two or more different classes of antiretroviral drugs, which can help reduce HIV-associated morbidity and mortality, and prevent transmission of the infection [1].

International guidelines recommend that patients not previously treated for HIV receive the nucleoside reverse transcriptase inhibitors emtricitabine and tenofovir disoproxil fumarate combined with a third medication: one of the ritonavir-boosted protease inhibitors (atazanavir or darunavir); the integrase inhibitor raltegravir; or the nonnucleoside reverse transcriptase inhibitor efavirenz [2].

Until recently, only one of these preferred regimens was formulated into a single tablet with efavirenz, emtricitabine and tenofovir disoproxil fumarate (EFV/FTC/TDF), marketed as Atripla ${ }^{\circledR}$ (Gilead Sciences, Foster City, CA, USA). Clinical trials have shown this tablet to have high efficacy, ease of administration and safety. The regimen has thus become widely used and is considered a gold standard for current practice [3-6].
However, not all patients can tolerate Atripla since it can cause central nervous system (CNS) side effects, rash and hyperlipidemia $[5,6]$. It also may increase the risk of teratogenicity during pregnancy when administered during the first trimester $[7,8]$. Thus, the addition of the newly US Food and Drug Administration (FDA)-approved single tablet HIV therapy for treatment-naïve patients known as Stribild ${ }^{\circledR}$ (Gilead Sciences, Foster City, CA, USA) is a welcome development.

The Stribild single-tablet regimen contains emtricitabine and tenofovir disoproxil fumarate plus elvitegravir and cobicistat (EVG/COBI/ FTC/TDF), a pharmacokinetic enhancer, which prolongs the effect of elvitegravir. Known in clinical studies prior to approval as "the Quad," the new single-tablet regimen was approved by the FDA in August 2012.

The purpose of this review is to discuss the efficacy, safety outcomes and side effects of Stribild as seen in two randomized double-blind phase 3 clinical trials, particularly when compared to Atripla and the treatment regimen of ritonavir-boosted atazanavir (ATV/ RTV) (Norvir ${ }^{\circledR}$; AbbVie Inc., North Chicago, IL, USA) plus emtricitabine and tenofovir disoproxil fumarate (Truvada $^{\circledR}$; Gilead Sciences, Foster City, CA, USA) in treatment naïve HIV patients $[9,10]$.

The article will also provide current data on the efficacy of Stribild and its component medications, information on Stribild's side effects and perspective on which HIV patients might benefit from this new single-tablet regimen HIV medication.

The two randomized, double-blind phase 3 noninferiority clinical trials of Stribild, which were the basis for the medication's recent approval by the FDA, studied its use in 1,408 adult patients not previously treated for HIV. In the first clinical trial, Stribild was compared to 
Atripla over 48 weeks. The second phase 3 trial measured outcomes with Stribild compared to ATV/RTV and Truvada taken once daily, also over 48 weeks $[9,10]$.

The results of both trials indicated that Stribild had high efficacy in controlling viral load and good tolerability over 48 weeks. It was shown to be noninferior when compared to the two different current HIV treatments and in some cases, had a more favorable side-effect profile $[9,10]$.

In the phase 3 clinical trials, Stribild treatment resulted in fewer abnormal dreams, less dizziness, insomnia and rash than, for example, Atripla, but an increase in nausea was observed. Stribild also resulted in fewer cases of abnormal liver function than ATV/ RTV plus Truvada and had smaller median increases in fasting cholesterol concentrations. However, a greater increase in serum creatinine was seen with Stribild than with Atripla $[9,10]$.

Stribild is now the only FDA-approved single-tablet regimen HIV medication that is composed of an integrase-inhibitor-based therapy. It is a highly effective alternative therapy for treatment-naïve HIV patients, which provides clinicians with greater flexibility in prescribing medications, without sacrificing ease of use for patients.

\section{METHODS}

A MEDLINE search was performed using the key words "elvitegravir," "cobicistat," "emtricitabine," and "tenofovir disoproxil fumarate" to identify relevant articles for inclusion in this review. Two randomized, double-blind phase 3 trials of Stribild, containing EVG/COBI/FTC/TDF, were identified.
In the clinical trials, outcomes with Stribild were compared to those with two other recommended HIV drug regimens. In one clinical trial, Stribild was compared to the once-daily tablet Atripla. In the other trial, outcomes with Stribild were compared to those with the treatment regimen of ATV/RTV plus Truvada taken once daily.

Two papers that studied the molecular mechanisms of Stribild and its component medications, such as cobicistat, were also identified.

\section{Dosages, Mechanism of Action and Pharmacokinetics}

Stribild is available in tablets containing $150 \mathrm{mg}$ of elvitegravir, $150 \mathrm{mg}$ of cobicistat, $200 \mathrm{mg}$ of emtricitabine and $300 \mathrm{mg}$ of tenofovir disoproxil fumarate. The recommended dosage is one tablet daily taken orally with food [11].

Studies on the pharmacokinetics and bioavailability of Stribild indicate that the use of cobicistat as a pharmacoenhancer results in high elvitegravir blood concentrations similar to those achieved with ritonavir-boosted elvitegravir [12, 13]. In a study by German et al. [13] in 2010, researchers also found that Stribild produced clinical exposures to tenofovir and emtricitabine that were equivalent to those seen with coadministration of these single agents, specifically emtricitabine $200 \mathrm{mg}$ capsules plus tenofovir $300 \mathrm{mg}$ tablets.

In this study of 42 subjects, patients were randomized to Stribild or ritonavir-boosted elvitegravir plusTruvada. The results indicated that elvitegravir systemic exposure and maximum concentrations of this drug were similar with Stribild than with ritonavirboosted elvitegravir plus emtricitabine/ tenofovir. Yet, trough concentrations of elvitegravir were lower [13]. 
More importantly tenofovir systemic exposure was similar in both treatment groups but maximum concentrations and trough concentrations were 30 and $24 \%$ higher with Stribild [13]. Although findings from initial clinical trials show that tenofovir is safe, there are case reports and observational studies that indicate it can be potentially nephrotoxic [14]. Stribild is now recommended only for patients with normal renal function, and those on the medication should have their renal function monitored regularly [14].

Given these pharmacokinetics, the fixed dose regimen of Stribild may be a favorable alternative to protease inhibitor-containing regimens associated with dyslipidemia and gastrointestinal adverse events. Stribild may also be a good choice for patients who are unable or unwilling to tolerate the efavirenzrelated CNS side effects seen with Atripla or for women of childbearing potential wishing to avoid the use of a pregnancy category D medication.

As with labels of many other drugs used to treat HIV, Stribild's label contains a boxed warning stating that the drug can cause a build-up of lactic acid in the blood and liver problems. The warning also notes that hepatic function should be monitored closely in patients receiving the drug, and should not be initiated in patients with estimated creatinine clearance $<70 \mathrm{~mL} / \mathrm{min}$. The drug is not approved to treat chronic hepatitis $\mathrm{B}$ virus infection. It is also not approved for those aged $<18$ years [11].

\section{Pivotal Phase 3 Clinical Trials of Stribild}

Two randomized double-blind phase 3 clinical trials have tested Stribild against other HIV medications including the once-daily HIV combination tablet, Atripla, now the gold standard for initial HIV treatment, and the regimen of ATV/RTV and taken with Truvada $[9,10]$.

Results indicated that Stribild had good efficacy outcomes as measured by viral suppression or decline in HIV ribonucleic acid (RNA) concentration, increase in CD4 cell count, and virological failure. Both clinical trials showed that Stribild had a good tolerability profile. The numbers of adverse events leading to drug discontinuation was similar for Stribild when compared to Atripla (4 vs. 5\% for Stribild and Atripla, respectively). In the study on Stribild and the regimen of ATV/RTV plus Truvada, Stribild had a favorable safety profile. In that study, $3.7 \%$ of patients on Stribild discontinued treatment because of side effects compared to $5.1 \%$ on ATV/RTV plus Truvada $[9,10]$.

\section{Efficacy Outcomes: Viral Suppression and CD4 Cell Count}

In the phase 3 clinical trial in which Stribild was compared to Atripla, 700 HIV patients from outpatient clinics in North America were randomized to one of the two treatments. All patients were adults diagnosed with HIV who had not received previous antiretroviral treatment and had plasma HIV RNA concentrations of 5,000 copies $/ \mathrm{mL}$ or higher. Laboratory samples and clinical data were regularly collected up to week 48 .

Results of the trial indicated that 305 of 348 patients $(87.6 \%)$ on Stribild versus 296 of 352 patients (84.1\%) on Atripla had an HIV RNA concentration of $<50$ copies $/ \mathrm{mL}$ at week 48 , which met the criteria for noninferiority [10]. Mean increases in CD4 cell count were also similar in both groups. However, patients on Stribild appeared to fare better by week 48 , with an increase of 239 versus 206 cells/ $\mu \mathrm{L}$ among 
Atripla patients $(P=0.009) . \quad$ Virological resistance in both groups was infrequent [10].

Virological suppression in this phase 3 trial was more rapid with Stribild than with Atripla. Up to week 16, a greater number of patients on Stribild than on Atripla achieved HIV RNA concentrations $<50$ copies $/ \mathrm{mL}$, although after that time point, response rates did not differ between the two drugs. High early response rates are typically seen with drugs in the integrase strand transfer inhibitor class, the investigators commented in their paper. "Although both CD4 cell response at week 48 and initial reduction in HIV RNA concentration are significantly greater with treatments based on strand-transfer integrase inhibitors compared with those based on efavirenz, the clinical significance of these differences is unknown" [10].

Of patients who received treatment, $4 \%$ $(n=31)$ met the criteria for resistance testing. Of 14 patients tested in the Stribild group, eight had resistance mutations. These eight patients had nucleoside reverse transcriptase inhibitor resistance mutations that included Met184Val/Ile and Met184Val/Ile plus Lys65Arg. Seven of eight also had primary integrase resistance mutations (primarily Glu92Gln [E92Q]).

Among the 17 patients in the Atripla group tested for resistance mutations, eight developed resistance to one or more components of the drug. The most common resistance profile was the Lys103Asn (K103N) mutation. Compared with Stribild, fewer patients on Atripla (2 vs. 8) had nucleoside/nucleotide reverse transcriptase inhibitor mutations (Met184Val/Ile with or without Lys65Arg) [10].

In a second randomized, double-blind phase 3 trial, Stribild was compared to another preferred initial therapy for HIV: the protease inhibitor regimen of ATV/RTV plus Truvada. All medications were taken once daily. Patients in both arms of the study each received four pills, similar in appearance, that were either active or placebo for Stribild, atazanavir, ritonavir, and emtricitabine/tenofovir disoproxil fumarate. In the trial, 718 randomly assigned patients from 146 sites in Australia, Europe, North America, and Thailand were treated with either regimen. The study population consisted of adults with HIV who had no previous antiretroviral treatment, and had plasma HIV RNA concentrations $\geq 5,000$ copies/. Patients in the trial also had to have a glomerular filtration rate (GFR) estimated to be at least $70 \mathrm{~mL} / \mathrm{min}$. Patients with hepatitis B or C coinfection were allowed to enroll. Five patients in the Stribild group and seven in the ATV/RTV plus Truvada were positive for hepatitis $\mathrm{B}$, and 18 in the Stribild arm and 10 in the ATV/RTV plus Truvada were positive for HCV antibody [9].

Results indicated that Stribild was noninferior to ATV/RTV plus Truvada after 48 weeks; 316 patients or $89.5 \%$ in the Stribild arm versus 308 or $86.8 \%$ in the ATV/RTV plus Truvada arm achieved an HIV RNA concentration of $\leq 50$ copies $/ \mathrm{mL}$. Patients on Stribild reached viral suppression earlier, with greater response rates up until week 16. After that time point, response rates did not differ between the two arms of the study [9].

CD4 cell counts rose to a similar extent in both groups. After 48 weeks, the mean CD4 cell count in the Stribild group had risen to 207 versus 211 cells $/ \mu \mathrm{L}$ in the ATV/RTV plus Truvada group. Development of resistance was infrequent in both groups. The researchers found that 'viral suppression rates in both groups (in the study) are among the highest reported in clinical trials of first treatment of adults with HIV infections, especially for regimens based on a protease inhibitor with ritonavir' [9]. 
The authors also noted [9] 'patients in the study had higher baseline CD4 cell counts than in earlier clinical trials of treatment naïve HIV patients, which might possibly be the result of new healthcare and HIV treatment guidelines.'

\section{Adverse Events in Clinical Trials}

The randomized double-blind phase 3 clinical trials on Stribild highlighted some of its advantages in terms of side effects, but also cautions for its use.

In the phase 3 clinical trial that measured the effects of Stribild versus Atripla on treatment naïve HIV patients, those receiving Stribild were less likely to have the CNS effects of abnormal dreams, dizziness and insomnia as well as rash. Although most of these adverse events were mild, some patients suffered moderate and severe abnormal dreams and dizziness, and these more serious side effects were less common in the Stribild group (two patients or $1 \%$ vs. 13 patients or $4 \%$ in the Atripla group, $P=0.007$ ) [10].

Nausea was significantly more common among Stribild users than among those on Atripla. However, nausea was generally mild and led to discontinuation in only one patient. The percentage of those experiencing moderate and severe nausea did not differ between the two groups (3\% in both arms) [10].

Stribild was shown to have significantly less effect on cholesterol levels than Atripla. Median fasting total cholesterol concentrations increased significantly less from baseline to week 48 in the Stribild group than in patients taking Atripla $(0.25 \mathrm{mmol} / \mathrm{L}$ vs. $0.49 \mathrm{mmol} / \mathrm{L}$; $P<0.001)$. Also, increase in low-density lipoprotein (LDL) concentrations was significantly less in the Stribild group than in the Atripla group $(0.26 \mathrm{mmol} / \mathrm{L}$ vs. $0.44 \mathrm{mmol} /$ L; $P=0.001)$, and there was significantly less reduction in high-density lipoprotein (HDL) cholesterol with Stribild $(0.13 \mathrm{mmol} / \mathrm{L}$ with Stribild vs. $0.20 \mathrm{mmol} / \mathrm{L}$ with Atripla, $P=0.001)$. Yet changes in the ratio of total to HDL cholesterol and increases in triglyceride levels were similar in the two treatment groups [10].

The major caution for use of Stribild is its effect on renal function; therefore regular monitoring for these effects in patients on the drug is crucial. In the phase 3 trial of Stribild versus Atripla for initial HIV treatment, five patients in the Stribild arm had renal adverse events that led to discontinuation, including two patients with increased serum creatinine, two with renal failure and one with Fanconi syndrome-all in the Stribild arm. However, the serum creatinine of one of these patients normalized within 2 weeks after stopping the drug [10].

The other four patients who suffered renal adverse events that led to discontinuation also developed signs of tubular toxicity (with a combination of glycosuria, proteinuria or hypophosphatemia). All these patients had signs of renal impairment before starting the study. Two had proteinuria. Two had a GFR $<70 \mathrm{~mL} / \mathrm{min}$ at baseline, but were allowed to participate in the study because the eGFR (estimated GFR) at their earlier screening visit

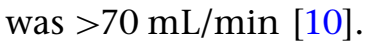

In the clinical trial, which assessed outcomes with Stribild versus those with ATV/RTV plus Truvada, discontinuation from either study drug was infrequent. Renal adverse events were also infrequent and only one patient in each treatment group discontinued their medication because of renal side effects. These side effects, which included increased creatinine concentration and toxic nephropathy, reversed after discontinuation of the study drugs [9]. 
Those on Stribild were also less likely to have increased alanine aminotransferase concentrations (54 patients or $15.3 \%$ vs. 76 patients or $21.6 \%$ in the ATV/RTV plus Truvada group) [9].

Those with clinically significant liver function test abnormalities at week 48 generally had underlying hepatic disease such as chronic hepatitis or a history of alcoholism, according to the researchers [9].

In a commentary that accompanied publication of the two trials, it was noted that the two published phase 3 trials on Stribild show that it has high efficacy as well as a good tolerability profile. However, they noted that Stribild's limitations include the potential for drug interactions and the need to be taken with food [14].

\section{Stribild in Clinical Practice}

An advantage of Stribild is the fact that it is a category B (unlike Atripla, which is a category D) medication in pregnancy and can potentially be prescribed to women of childbearing age. When compared to other single-tablet regimen treatments for initial HIV therapy, Stribild has another advantage in that it can be prescribed for patients with high viral load. This is in contrast to the FDA label for Complera ${ }^{\circledR}$ (Gilead Sciences, Foster City, CA, USA), which contains emtricitabine/rilpivirine/tenofovir disoproxil fumarate, and is not recommended for people with a viral load of $>100,000$.

Stribild can be particularly useful in women of childbearing age with a viral load of over 100,000, who express a preference for a single-tablet regimen therapy. Other considerations when prescribing Stribild are the patient's renal function. A personal and family history of hyperlipidemia and personal or family history of heart disease would make Stribild the drug of choice over Atripla because of its benefits in terms of lipid profile. However, Stribild would not be drug of choice where there is evidence of any hepatic disease or compromised renal function.

\section{CONCLUSION}

With 27 antiretroviral drugs now approved in the United States, including Stribild, HIV treatment can now be individualized to each patient. There has also been a great deal of progress in reducing the pill burden of combination regimens for HIV patients, which has increased adherence. Adherence and high efficacy are now important cornerstones for HIV treatment and prevention of new cases.

The addition of Stribild to HIV treatment options for newly diagnosed patients provides us with an important choice. It is a Category B pregnancy drug, providing a choice for women of childbearing age. It is also gentler on cholesterol than other combination single-tablet regimens for HIV. It has the advantage of being approved without special considerations for those with a viral load of over 100,000.

The success of Atripla, Complera and Stribild will no doubt inspire the generation of more single-tablet regimens for HIV-a scenario that will be welcomed by both clinicians and patients. When even more single-tablet regimens become available, clinicians will have a greater number of efficacious and convenient treatment options for their patients. As well as providing a range of choices for patients in terms of their side-effect profile, these therapies can be easily incorporated into a modern lifestyle. Stribild offers comparable efficacy to two US Department of Health and Human Services Guideline preferred regimens, Atripla and ATV/ RTV with Truvada, with fewer CNS side effects and better cholesterol changes than Atripla. It also causes less bilirubin and triglyceride 
changes than the boosted atazanavir regimens. For childbearing women or for those who cannot tolerate the CNS effects of Atripla, Stribild is an especially advantageous choice.

\section{ACKNOWLEDGMENTS}

Dr. Cynthia Brinson is the guarantor for this article, and takes responsibility for the work as a whole.

Conflict of interest. Cynthia Brinson is on the Speaker's Bureau for Gilead Sciences.

Open Access. This article is distributed under the terms of the Creative Commons Attribution Noncommercial License which permits any noncommercial use, distribution, and reproduction in any medium, provided the original author(s) and the source are credited.

\section{REFERENCES}

1. US Department of Health and Human Services Panel on Antiretroviral Guidelines for Adults and Adolescents. Guidelines for the use of antiretroviral agents in HIV-1 infected adult and adolescents. 2012; Department of Health and Human Services. http://aidsinfo.nih.gov/contentfiles/lvguidelines/ adultandadolescentgl.pdf. Accessed February 14 2013.

2. Thompson MA, Aberg JA, Cahn P, et al. Antiretroviral treatment of adults with HIV infection. Recommendations of the International AIDS Society-USA Panel. JAMA. 2010;2010(304): 321-33.

3. Arribas JR, Pozniak AL, Gallant JE, et al. Tenofovir disoproxil fumarate, emtricitabine, and efavirenz compared with zidovudine/lamivudine and efavirenz in treatment-naive patients; 144-week analysis. J Acquir Immune Defic Syndr. 2008;47: 74-8.

4. Sax PE, Tierney C, Collier AC, et al. Abacavirlamivudine versus tenofovir-emtricitabine for initial HIV therapy. N Engl J Med. 2009;361: 2230-40.
5. Cohen CJ, Andrade-Villanueva J, Clotet B, et al. Rilpivirine versus efavirenz with two background nucleoside or nucleotide reverse transcriptase inhibitors in treatment-naïve adults infected with HIV-1 (THRIVE): a phase 3 randomised, noninferiority trial. Lancet. 2011;378:229-37.

6. Molina JM, Cahn P, Grinsztejn B, et al. Rilpivirine versus efavirenz with tenofovir and emtricitabine in treatment-naïve adults infected with HIV-1 (ECHO): a phase 3 randomised double-blind active-controlled trail. Lancet. 2011;378:238-46.

7. Lennox JL, DeJesus E, Lazzarin A, et al. Safety and efficacy of raltegravir-based versus efavirenz-based combination therapy in treatment-naive patients with HIV-1 infection: a multicentre, double-blind randomised controlled trail. Lancet. 2009;374: 796-806.

8. ATRIPLA (efavirenz/emtricitabine/tenofovir disoproxil fumarate) [U.S. prescribing information]. Foster City: Gilead Sciences and Bristol-Myers Squibb; 2012.

9. DeJesus E, Rockstroh JK, Henry $\mathrm{K}$, et al. Coformulated elvitegravir, cobicistat, emtricitabine, and tenofovir disoproxil fumarate versus ritonavirboosted atazanavir plus co-formulated emtricitabine and tenofovir disoproxil fumarate for initial treatment of HIV-1 infection: a randomised, double-blind, phase 3 , non-inferiority trial. Lancet. 2012;379:2429-38.

10. Sax PE, DeJesus E, Mills A, et al. Co-formulated elvitegravir, cobicistat, emtricitabine, and tenofovir versus co-formulated efavirenz, emtricitabine, and tenofovir versus co-formulated efavirenz, emtricitabine, and tenofovir for initial treatment of HIV-1 infection: a randomised, double-blind, phase 3 trial, analysis or results after 48 weeks. Lancet. 2012;379:2439-48.

11. STRIBILD (elvitegravir, cobicistat, emtricitabine, tenofovir disoproxil fumarate) [FDA package insert]. Foster City: Gilead Sciences Inc.; 2012.

12. El Lepist, Phan TK, Roy A, et al. Cobicistat boosts the intestinal absorption of transport substrates, including HIV protease inhibitors and GS-7340, in vitro. Antimicrob Agents Chemother. 2012;56:5409-13.

13. German P, Warren D, West S, Hui J, Kearney BP. Pharmacokinetics and bioavailability of an integrase and novel pharmacoenhancercontaining single-tablet fixed-dose combination regimen for the treatment of HIV. J Acquir Immune Defic Syndr. 2010;55:323-9.

14. Schrijvers R, Deyser Z. Quad's in it for antiretroviral therapy? Lancet. 2012;379:2403-4. 